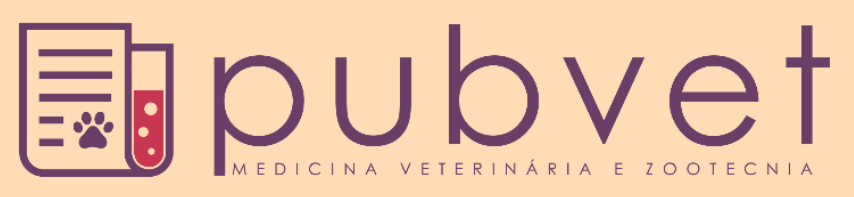

https://doi.org/10.31533/pubvet.v12n12a224.1-5

\title{
Hiperplasia endometrial e hematometra associadas ao adenocarcinoma ovariano em cadela Submetida a OSH terapêutica: Relato de caso
}

\author{
Liliane Cristina Jerônimo dos Santos ${ }^{1 *}$, João Iribarrem $\operatorname{Vargas}^{1}{ }^{\bullet}$, Tainá Ança Evaristo ${ }^{1}$,

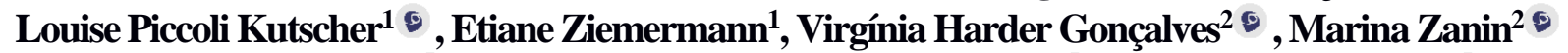 \\ Bruna dos Santos Pires $^{3 \oplus}$, Fabrício de Vargas Arigony Braga ${ }^{4}{ }^{\bullet}$, Patrícia Silva Vives ${ }^{5}$ \\ ${ }^{I}$ Acadêmicos de Medicina Veterinária, Universidade Federal de Pelotas, Pelotas/RS-Brasil; \\ ${ }^{2}$ Médica veterinária, autônoma, Pelotas/RS Brasil; \\ ${ }^{3}$ Residente em Clínica Médica Cirúrgica de Animais de Companhia, Universidade Federal de Pelotas, pelotas/RS Brasil; \\ ${ }^{4}$ Docente, Departamento de Clínicas Veterinárias, Universidade Federal de Pelotas, pelotas/Rs Brasil; \\ ${ }^{5}$ Veterinária da área de Clínica Médica Cirúrgica de Animais de Companhia, Departamento de Clínicas Veterinárias, \\ Universidade Federal de Pelotas, Pelotas/RS Brasil. \\ * Autor para correspondência, E-mail: liliane.c.d.j@gmail.com
}

\begin{abstract}
RESUMO. O adenocarcinoma ovariano representa $1,2 \%$ de todas as neoplasias diagnosticadas em cadelas, afeta um ou ambos os ovários e pode ser encontrado como achado durante ovário-histerectomias eletivas. Em cães, este neoplasma surge a partir de invaginações das estruturas de subsuperfícies epiteliais na cápsula do ovário. Porém, essa enfermidade apresenta-se pobremente descrita na literatura. Desse modo, objetiva-se relatar o caso de uma cadela com 13 anos de idade, apresentando anemia, azotemia, secreção vaginal sanguinolenta e diagnóstico presuntivo de hematometra/piometra por meio de ecografia abdominal. A paciente foi estabilizada e submetida a ovariohisterectomia terapêutica. O diagnóstico histopatológico revelou adenomiose uterina, hiperplasia glandular não cística e adenocarcinoma ovariano subtipo papilar. Após o procedimento cirúrgico a paciente foi acompanhada por cinco meses e apresentou completa remissão dos sinais clínicos sem evidências de metástase.
\end{abstract}

Palavras chave: adenomiose uterina, anemia, neoplasia, ovariohisterectomia

\section{Endometrial hyperplasia and hematometry associated with ovarian adenocarcinoma in female dog Submitted to ovario-hysterectomie: Case report}

\begin{abstract}
The ovarian adenocarcinoma represents $1.2 \%$ of diagnosed neoplasms in female dogs; affects one or both ovaries and can be a finding during elective ovariohysterectomies. In dogs, neoplasmas of the ovarian epithelium emerge from invaginations of epithelial subsurface structures in the ovary capsule. However, this disease is poorly described in the literature. Thus, it was aimed to report the case of a 13-year-olf-female dog showing anemia, azotemia, bloody vaginal secretion and hematometry/pyometra as presumptive diagnosis through abdominal echography. The patient was stabilized and was submitted to a therapeutic ovario-hysterectomy. The hystopathological diagnosis showed uterine adenomyosis, noncystic glandular hyperplasia and ovarian adenocarcinoma papillary subtype. After the surgical procedure, the patient was monitored for five months and showed complete remission of clinical signs with no evidence of metastasis.
\end{abstract}

Keywords: anemia, uterine adenomyosis, neoplasia, ovariohysterectomy. 


\title{
Hiperplasia endometrial y hematometra asociada al adenocarcinoma ovárico en perra Sometida a OSH terapéutica: Relato de caso
}

\begin{abstract}
RESUMEN. El adenocarcinoma ovárico representa el 1,2\% de todas las neoplasias diagnosticadas en perras, afecta a uno o ambos ovarios y puede ser encontrado como hallado durante las ovariohistencias automáticas electivas. En perros, estos neoplasmas del epitelio ovárico surgen a partir de invaginaciones de las estructuras de subsuperficies epiteliales en la cápsula del ovario. Sin embargo, esta enfermedad se presenta pobremente descrita en la literatura. De este modo, se objetiva relatar el caso de una perra con 13 años de edad, presentando anemia, azotemia, secreción vaginal sanguinolenta y diagnóstico presuntivo de hematometra/piometra por medio de ecografía abdominal. La paciente fue estabilizada y sometida a la ovariohisterectomía terapéutica. El diagnóstico histopatológico reveló adenomiosis uterina, hiperplasia glandular no quística y adenocarcinoma ovárico subtipo papilar. Después del procedimiento quirúrgico la paciente fue acompañada por cinco meses y presentó completa remisión de los signos clínicos sin evidencia de metástasis.
\end{abstract}

Palabras clave: adenomiosis uterina, anemia, neoplasia, ovariohisterectomía

\section{Introdução}

Os tumores ovarianos são divididos em três grupos de acordo com sua origem: neoplasmas epiteliais, de células germinativas e de estroma gonadal (Sforna et al. 2003). As espécies frequentemente acometidas são caninas e equinas com possibilidade de comprometimento de ambos os ovários, e também a espécie bovina (Souza et al. 2016).

As neoplasias ovarianas representam 1,2\% de todos os tumores em cadelas (Seoane 2010) e são compostas por múltiplos cistos contendo fluidos ou muco (Schlaffer \& Miller 2007) com elevado potencial metastático em linfonodos, rins, ovários, baço, entre outros órgãos (Johnston 1998). Os carcinomas ovarianos podem apresentar-se silenciosos ou com manifestação de sinais clínicos, como por exemplo, distensão da cavidade abdominal, efusões peritoneais, descargas vulvares e endocrinopatias, uma vez que alguns são hormonalmente ativos, produzindo esteroides, com evolução até o óbito (Yotov et al. 2005).

O objetivo do presente trabalho é descrever o tratamento clínico e cirúrgico de uma cadela com adenocarcinoma ovariano, associado a hematometra e piometra.

\section{Relato de caso}

Foi atendida em uma clínica particular da cidade de Pelotas, Rio Grande do Sul, uma cadela da raça Poodle, com 13 anos de idade, $5 \mathrm{~kg}$ de massa corporal, não castrada, com histórico de prostração, inapetência, polidipsia, secreção vaginal sanguinolenta e lambedura excessiva na região vulvar. Ao exame clínico foi constatado apatia, mucosas pálidas, aumento do volume abdominal, edema e secreção sanguinolenta vaginal. Exames laboratoriais evidenciaram hematócrito de $17 \%$, aumento discreto de bastonetes $(393 \mu \mathrm{L})$, creatinina $2,4 \mathrm{mg} / \mathrm{dL}$ e ureia $130,4 \mathrm{mg} / \mathrm{dL}$, demais análises dentro dos parâmetros fisiológicos.

A ecografia abdominal revelou útero ocupando grande parte da cavidade peritoneal, paredes espessadas e presença de conteúdo hipoecogênico, sugerindo piometra/hematometra (Figura 1A), parênquima hepático heterogêneo, hipoecogênico compatível com hepatopatia (Figura 1B) e rins apresentando aumento da ecogenicidade, sugestivo de glomerulonefrite (Fig. 1C).

Diante deste quadro a paciente foi submetida à transfusão sanguínea fracionada em três etapas: pré, trans e pós-operatória, totalizando $250 \mathrm{ml} \mathrm{de}$ sangue, elevando o hematócrito para $31 \%$, fluidoterapia para estabilização hemodinâmica, e ovariosalpingohisterectomia terapêutica.

Após preparo de rotina e plano anestésico adequado, o procedimento cirúrgico iniciou por meio de celiotomia mediana retroumbilical, a cavidade abdominal foi inspecionada e observouse um nódulo na parede do corno uterino esquerdo de aproximadamente $2 \mathrm{~cm}$, útero bastante distendido (Figura 2A) e cistos ovarianos (Figura 2B). 

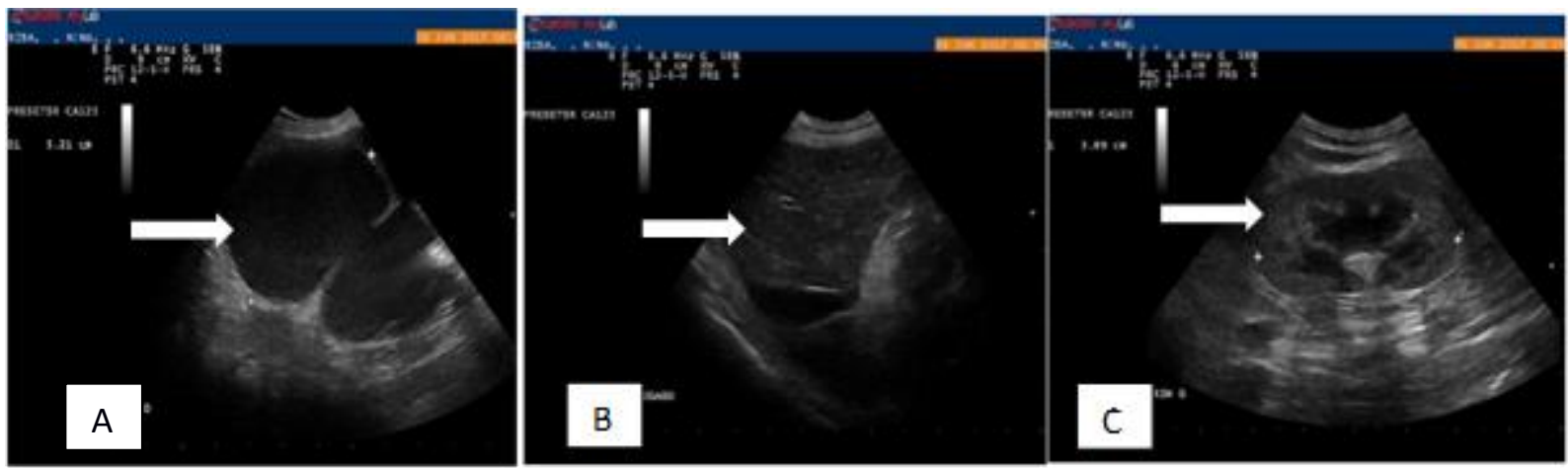

Figura 1. Ecografia abdominal de uma cadela poodle, 13 anos de idade com distensão abdominal demonstrando: (A) aumento uterino sugestivo de piometra/ hematometra (seta); (B) parênquima hepático heterogêneo, hipoecogênico compatível com hepatopatia (seta); (C) e aumento da ecogenicidade renal, sugestivo de glomerulonefrite (seta).

A ovariosalpingohisterectomia ocorreu por meio da técnica das 3 pinças modificada utilizando-se náilon monofilamentar 2-0 para as ligaduras e sutura de Parker-quer para o coto uterino, cobrindo-o e suturando uma pequena porção do omento maior. A celiorrafia ocorreu com sutura continua simples e fio de náilon monofilamentar 2-0, o subcutâneo foi reduzido com sutura continua simples e náilon monofilamentar 3-0 e a pele suturada com padrão intradérmico e náilon 3-0.

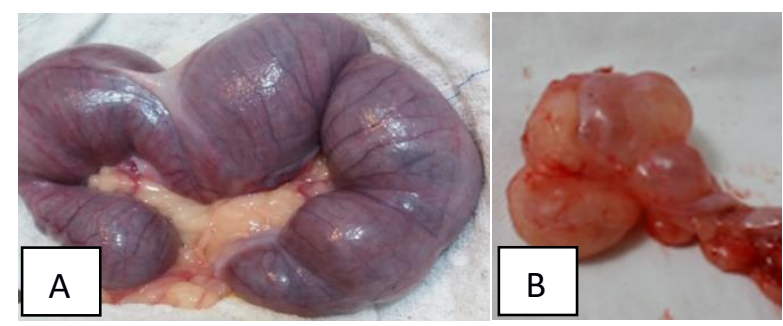

Figura 2. Imagens do transoperatório de uma cadela com aumento de volume abdominal submetida a ovariosalpingohisterectomia. (A) Exposição uterina evidenciando-se cornos uterinos distendidos. (B) Aspecto macroscópico nodular cístico do ovário direito, após ressecção cirúrgica.

Após o procedimento, útero e ovários foram encaminhados para análise histopatológica e apresentaram três diagnósticos: adenomiose uterina, hiperplasia glandular não cística e adenocarcinoma ovariano subtipo carcinoma papilar.

A paciente ficou internada por quatro dias recebendo fluidoterapia intravenosa, tramadol 4 $\mathrm{mg} / \mathrm{kg}$, TID, dipirona $25 \mathrm{mg} / \mathrm{kg}$, TID e sulfametoxazol/trimetoprin $20 \mathrm{mg} / \mathrm{kg}$, BID. Após esse período, obteve alta com manutenção das medicações por mais três dias e recomendação de retorno em 10 dias, para remoção dos pontos.

\section{Resultados e discussão}

Ao retorno em 10 dias, a cadela estava plenamente recuperada. $\mathrm{O}$ animal ficou sob observação por quatro meses, sem sinais clínicos ou surgimento de metástases no período acompanhado.

$\mathrm{Na}$ hematometra ocorre diapedese de eritrócitos para o lúmen uterino, ocasionando consequentemente a depressão tóxica da medula óssea e inibição da eritropoiese (Trindade et al. $\underline{2010}$ ), fato este que justifica a presença de anemia da paciente. A transfusão realizada em pacientes anêmicos na forma de sangue total ou na forma de concentrado de eritrócitos tem também como finalidade melhorar o transporte de oxigênio para os tecidos por meio do aumento da concentração de hemoglobina sanguínea (Prittie 2010).

A elevação sérica de bastonetes é um sinal de requerimento de neutrófilos nos tecidos durante o processo inflamatório, já concentrações séricas de ureia e creatinina aumentadas podem estar relacionadas à reduzida perfusão renal e/ou à toxemia. Esta toxemia leva ao aumento do catabolismo proteico, podendo aumentar as concentrações de creatinina (Verstegen et al. 2008).

A hematometra é caracterizada por secreção vaginal hemorrágica, relacionada com neoplasia vaginal e uterina, lacerações, hiperplasia endometrial ou torção uterina (Sampaio et al. 2002). Além disso, também pode ser uma consequência da piometra pela diapedese de eritrócitos para a luz uterina, a qual modifica a coloração da secreção (Trindade et al. 2010). A terapia deve ser imediata e agressiva e a ovariohisterectomia é considerada o tratamento de eleição nesses casos para resolução definitiva, garantindo o sucesso do tratamento (Trindade et al. 2010). 
A adenomiose uterina consiste na presença de tecido endometrial no interior do miométrio em que macroscopicamente o útero sofre um aumento simétrico difuso, conforme observado na paciente relatada, ou assimétrico focal (Foster 2013). Acomete com menor frequência cadelas e gatas e ocorre em fêmeas de meia idade a idosas, sem predisposição racial, sendo a maioria encontrada como achado acidental na necropsia (Fossum 2014). A adenomiose uterina, geralmente, está associada a outros distúrbios uterinos, como piometra, hematometra, endometrites e hiperplasia endometrial cística (Prestes et al. 1997)). A hiperplasia glandular não cística não é macroscopicamente reconhecível e, frequentemente, está associada à adenomiose uterina (Foster 2013).

$\mathrm{O}$ adenocarcinoma ovariano é uma neoplasia incomum e pode se apresentar de forma sólida, cística, séssil e/ou polipóide podendo obliterar o lúmen uterino, resultando em piometra/hematometra (Fossum 2014). A baixa ocorrência pode estar relacionada à prática comum da cirurgia precoce de ovariohisterectomia (Félix et al. 2015).

Grande parte das cadelas que desenvolvem estes tumores apresenta hiperplasia endometrial cística (Foster 2013) e normalmente são assintomáticas, porém, se houver grandes massas, essas podem comprimir os sistemas urinário $\mathrm{e}$ gastrointestinal. O diagnóstico é realizado a partir da anamnese, exames físicos e laboratoriais, radiografia e ultrassonografia abdominal (Volpato et al. 2012).

De acordo com o caso relatado e a literatura citada, provavelmente o adenocarcinoma foi a doença primária dentre os três diagnósticos encontrados na análise histopatológica, sendo que este desencadeou as demais doenças agravando o quadro clínico do animal.

\section{Conclusão}

O diagnóstico precoce e a terapia de suporte prévia a ovariosanpingohisterectomia foram decisivas para a recuperação plena desta paciente e o diagnóstico histopatológico foi fundamental para compreender a dinâmica destas enfermidades.

\section{Referências bibliográficas}

Félix P.G., Seixas G., Oliveira L.R.R. \& Silva T. 2015. Tumor de células da granulosa em cadela: Relato de caso. Revista de Educação
Continuada em Medicina Veterinária e Zootecnia do CRMV-SP 13, 97-97.

Fossum T.W. 2014. Cirurgia de pequenos animais. Elsevier Brasil, São Paulo.

Foster R.A. 2013. Sistema Reprodutivo da Fêmea. In: Bases da Patologia Veterinária (eds. by McGavin MD \& Zachary JF). Elsevier, Rio de Janeiro, Brasil.

Johnston S.D. 2098. Oncologia - Sistemas Reprodutivos. In: Manual de Cirurgia de Pequenos Animais (ed. by D. S). Manole, São Paulo, Brasil.

Prestes N.C., Bicudo S.D., Landin F.C., Sartori Filho R. \& Castilho C. 2097. Aplasia segmentar de um corno uterino associada à piometra em cadela. Veterinária Notícias Veterinary News 3, 133-134.

Prittie J.E. 2010. Controversies related to red blood cell transfusion in critically ill patients. Journal of Veterinary Emergency and Critical Care 20, 167-176.

Sampaio R.L., Sampaio R. \& Lacerda M.S. 2002. Ruptura de útero em cadelas gestantes após a aplicação de ocitocina: relato de 2 casos. In: Congresso Brasileiro de Medicina Veterinária (Conbravet), Gramado, Rio Grande do Sul.

Schlaffer D.H. \& Miller R.B. 2007. Female Genital System. In: Pathology of domestic animals (ed. by Maxie MG). Academic Press, San Diego, USA.

Seoane M.P.R. 2010. Avaliação da ocorrência das alterações ultrassonográficas na cavidade abdominal, detectadas em cães idosos clinicamente saudáveis atendidos no Hospital Veterinário da Universidade Federal do Paraná. In: Faculdade de Veterinária, p. 112 p. Universidade Federal do Paraná, Curitiba, Paraná, Brasil.

Sforna M., Brachelente C., Lepri E. \& Mechelli L. 2003. Canine ovarian tumours: a retrospective study of 49 cases. Veterinary research communications 27, 359-361.

Souza N.F., Silveira K.F. \& Aguirra M.V.R.L. 2016. Adenocarcinoma ovariano em cadela. Revista Brasileira de Reprodução Animal 40, 516-517.

Trindade A.B., Brun M.V., Basso P.C., Oliveira N.F., Bertoletti B., Bortolini C.E. \& Contesini E.A. 2010. Ovário-histerectomia videoassistida em uma cadela com hematometra. Ciência Animal Brasileira 11, 228-233. 
Verstegen J., Dhaliwal G. \& Verstegen-Onclin K. 2008. Mucometra, cystic endometrial hyperplasia, and pyometra in the bitch: advances in treatment and assessment of future reproductive success. Theriogenology 70, 364374.

Volpato R., Salgado B.S. \& Leardini E.G. 2012. Carcinoma papilar ovariano em cadela relato de caso. Clínica Veterinária 17, 6056-6056.

Yotov S., Simeonov R., Dimitrov F., Vassilev N., Dimitrov M. \& Georgiev P. 2005. Papillary ovarian cystadenocarcinoma in a dog: clinical communication. Journal of the South African Veterinary Association 76, 43-45.

Recebido: 16 setembro, 2018 .

Aprovado: 30 outubro, 2018.

Publicado: 26 dezembro, 2018.

Licenciamento: Este artigo é publicado na modalidade Acesso Aberto sob a licença Creative Commons Atribuição 4.0 (CC-BY 4.0), a qual permite uso irrestrito, distribuição, reprodução em qualquer meio, desde que o autor e a fonte sejam devidamente creditados. 